Article

\title{
Challenges and Opportunities for Integrating In Silico Models and Adverse Outcomes Pathways to Set and Relate New Biomarkers
}

\author{
Carlos E. Matos dos Santos ${ }^{1,2, * \mathbb{D}}$, Raul Ghiraldelli Miranda ${ }^{3}{ }^{(\mathbb{C}}$, Danielle Palma de Oliveira ${ }^{1}$ \\ and Daniel Junqueira Dorta ${ }^{3}$ (i) \\ 1 School of Pharmaceutical Sciences of Ribeirão Preto, University of São Paulo, \\ Ribeirão Preto, SP 14040-903, Brazil; dpalma@usp.br \\ 2 Altox Ltd., Brazil-Alternative Toxicology, São Paulo, SP 05503-001, Brazil \\ 3 Department of Chemistry, Faculty of Philosophy, Sciences and Letters of Ribeirão Preto, \\ University of São Paulo, Ribeirao Preto, SP 14040-903, Brazil; raul.miranda@usp.br (R.G.M.); \\ djdorta@ffclrp.usp.br (D.J.D.) \\ * Correspondence: carlosematos@usp.br
}

Received: 21 October 2020; Accepted: 4 December 2020; Published: 17 December 2020

\begin{abstract}
The Adverse Outcome Pathway (AOP) framework has been considered the most innovative tool to collect, organize, and evaluate relevant information on the toxicological effects of chemicals, facilitating the establishment of links between molecular events and adverse outcomes at the critical level of biological organization. Considering the combination of the high volume of toxicological and ecotoxicological data produced and the application of artificial intelligence algorithms from the last few years, not only can higher mechanistic interpretability be reached with new in silico models, but also a potential increase in predictivity in hazard assessments and the identification of new potential biomarkers can be achieved. The current paper aims to discuss some potential challenges and ways of integrating in silico models and AOPs to predict toxicological effects and to set and relate new biomarkers for defined purposes. With the use of the AOP framework to organize the ecotoxicological, toxicological, and structural data generated from in chemico, in vitro, ex vivo, in vivo, and population studies, it is expected that the generated biological and chemical construct will improve its application, establishing a knowledge platform to set and relate new biomarkers by key event relationships (KERs).
\end{abstract}

Keywords: Adverse Outcome Pathway; in silico models; biomarkers; predictive toxicology

\section{Introduction}

In Silico Toxicology (IST) integrates different mathematical models to predict the toxicity of chemicals based on patterns of structural and physicochemical properties related to the toxicological activity.

With increasing relevance in different applications and as a cost-effective tool with a potentially higher mechanistic interpretability in evidence-driven assessments, in silico models (computer-based) are considered useful for predicting the toxicity of chemicals with unknown biological activity and are recommended by regulators and/or proposed by investigators for use in various contexts. Some types of substances assessed by in silico models in the last few years include drug impurities and degradation products [1]; pesticide metabolites and impurities [2]; additives, neo-formed contaminants (NFCs), and contaminants in food [3-5]; cosmetic ingredients [6]; industrial chemicals and emerging chemicals (ECs) [7]; and others. 
There are several limitations of animal testing methods beside the ethical and economical concerns [8], and it is a challenge to define when there is sufficient confidence or predictivity level to use one or more alternative models for a defined purpose.

Regarding the combination of the high volume of toxicological and ecotoxicological data produced in the last few years and the advances in new powerful techniques and artificial intelligence algorithms, not only can mechanistic interpretability be reached with in silico models (e.g., alert-based models), but so also can a potential increase in predictivity in hazard assessments and the identification of new potential biomarkers.

Despite significant development in these computational models and the increased availability of new experimental data from traditional and alternative methods, frequently there have been doubts about how the different kinds of in silico models generated by these data should be applied and how to integrate these predictions with in chemico, in vitro, ex vivo, in vivo, and population data for defined purposes.

Over the last few decades, some systems- and pathway-based approaches have been discussed by scientific groups to drive these topics. In 2013, the Organization for Economic Co-operation and Development (OECD) published the first version of the Guidance Document on Developing and Assessing Adverse Outcome Pathways with a conceptual background [9], followed by the publication of the Users' Handbook Supplement in 2018 [10], containing more practical instructions for Adverse Outcome Pathway (AOP) development and collaborative work on the databases AOP knowledgebase (AOP-KB) (http://aopkb.org/) and AOP-Wiki (https://aopwiki.org).

The current paper aims to discuss some potential challenges and ways of integrating in silico models and AOP to predict toxicological effects and assess potential relationships between biomarkers.

\section{Adverse Outcomes Pathways (AOPs) as a Framework to Set New Biomarkers}

The absence of links between ecotoxicological and toxicological outcomes and specific biomarkers is one of the major challenges for applying some biomarkers in regulatory decision-making or to define management objectives [11].

The AOP methodology provides a framework to collect, organize, and evaluate relevant information on the biological and toxicological effects of chemicals, establishing links between the molecular-level disruption of a biological system and an adverse outcome at a level of biological organization of regulatory concern [9]. This approach supports the use of a mode or mechanism of action basis and a sequential chain of causally linked key events (KEs) at different levels of biological organization that lead to adverse outcomes (AOs) (Figure 1).
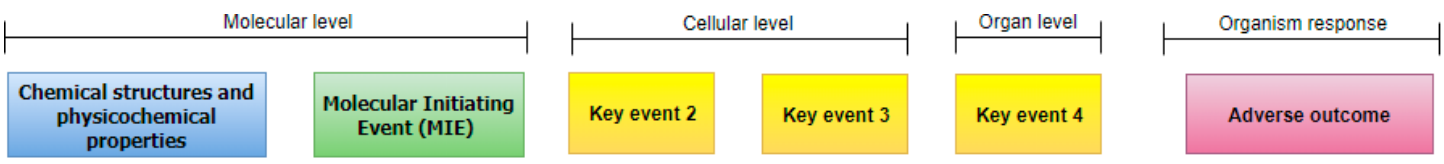

Figure 1. Representation of an Adverse Outcome Pathway (AOP) and its modular components.

By definition, a molecular initiating event (MIE) is a specialized type of key event that represents the initial point of chemical interaction at the molecular level within the organism that results in a perturbation that starts the AOP. A KE is a change in biological state that is both measurable and essential to the progression of a defined biological perturbation leading to a specific $\mathrm{AO}$ [9]. An $\mathrm{AO}$ is a specialized KE that represents the end (an adverse outcome of regulatory significance) of an AOP. Based on the OECD's Handbook Supplement, for KEs that are designated as an AO, additional fields of information (regulatory significance of the AO) should be provided [10].

The AOP framework is composed of a set of modular components (Figure 1). At the molecular level, relevant mechanistic data are considered, including the structural and physicochemical properties of the chemical/or chemical categories and macromolecular interactions with a biomolecule or specific target (e.g., receptors, transport proteins, DNA binding, etc.), generally obtained by in silico, in chemico, 
and in vitro methods. At the cellular and organ levels, a sequence of key events potentially linked among each other is considered, generally including data obtained by organoids/mini-organs and $e x$ vivo or in vivo assays. Finally, at the organism or physiological level, an adverse outcome is considered. This framework can be expanded to population and/or community levels, and different biomarkers can be set for different key events along with the AOP.

As a challenge, there is a variable level of confidence, knowledge, and relationships between KEs at different AOPs. Based on the OECD criteria, each component of an AOP may itself be influenced by other pathways ongoing within the biological system being modeled, a Weight of Evidence (WoE) approach is proposed for judgment, and some parameters of assessment are established (essentiality, causality, and biological plausibility) [9].

Additionally, by exploring the AOP networks it may be possible to identify measurable KEs to be used as biomarkers of exposure or effect and their relationships by the Key Event Relationships (KERs) (Figure 2). Based on the OECD criteria, the essentiality parameter refers to experimental data for whether or not downstream KEs or the AO are prevented or modified if an upstream event is blocked.

Key Event Relationships (KERs)

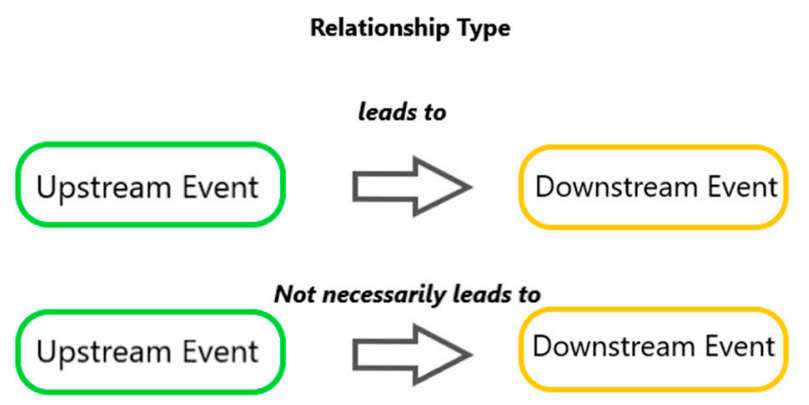

Figure 2. Some of the types of Key Event Relationships (KERs), showing the possible and theoretical relationships between an upstream key event and a downstream key event. Along with the AOP, the essentiality assessment could be used to identify biomarkers of effect or exposure useful to prevent or modify adverse pathways.

If a sequential chain of causally linked events is known or sufficiently elucidated, links between biomarkers of exposure or effect would be explored to design strategies or defined approaches for decision-making-for example, predicting a set of specific biomarkers that would be directly related or not, but useful to confirm the exposures or observed adverse outcomes. Patterns of multiple key events not necessarily related can be attributed to specific chemicals and explored from a wide biological perspective.

As a result, an AOP is a biological construct, structured and connected by modular components with a configuration of "biological space", to be explored about patterns and for the generation of information that is evidence-based and potentially able to be translated to computational language and explored by algorithms to generate new hypotheses.

The assessment of the acceptance of AOPs for application in different regulatory contexts is based on the confidence and precision levels with which the KEs can be measured, the plausibility and theoretical support, and the evidence for the overall pathway. As per the proposed vision for toxicology in the 21st century, there is a significant interest in extrapolating from in silico, in chemico, and in vitro results (based on low levels of biological organization) to outcomes at higher levels of biological organization considered relevant to regulatory protection goals and decision-making [10].

\section{Examples of AOP Uses in Studies of Biomarkers}

As stated before, a KE description contains information on the biological state, biological compartment, and KERs (key events relationships) that can provide a connection to AOs. This kind of description brings important information, because a KE that can be easily collected 
(in fluids like plasm, cerebrospinal fluids) with noninvasive procedures has more potential to become a biomarker than a KE that only can be measured in tissue [12].

Some examples of using AOP to retrieve, set, and relate biomarkers are described below for some adverse outcome, such as neurotoxicity, endocrine disruption, reproductive effect, DNA, and mutagenic effect, etc.

Spinu et al. (2019) proposed the development of an AOP network for human neurotoxicity using linear AOPs from the OECD AOP-Wiki 2.0 not only to develop and analyze AOP but also to identify the most common/highly connected KEs and KERs as a basis for quantitative modeling for neurotoxicity [13]. The authors established a workflow of four steps that can be applied and adapted according to the purpose of the investigation. The four steps consisted of (1) identifying the aim of the AOP network; (2) defining the criteria for AOP selection; (3) the identification of appropriated AOP based on the previously defined criteria; and, finally, (4) generating and analyzing the network using a computational tool. In this context, Spinu, et al. demonstrated the applicability of an AOP network generating data to prioritize the KEs to be tested and to identify neurotoxicity biomarkers that can be used in in silico models [13].

Baken et al., 2019, carried out another AOP strategy to validate human biomarkers of effect for reproductive outcomes after phthalate exposure [14]. For that, the published information on biomarker effects was combined with the results of experimental studies and already published AOPs for reproductive effects. Considering that, the authors found an extensive list of published biomarkers of effect and using AOPs, they could associate and identify candidates to new biomarkers that should be verified to their association to adverse health effects of specific or multiple phthalates. For examples, Peroxisome Proliferator-Activated Receptor (PPAR $\alpha$ ), PPAR $\gamma$, and Glucocorticoid receptor (GR) activation were identified as early events that can lead to a downstream event at the steroidogenic enzyme level and as a consequence can reduce testosterone levels and induce male reproductive tract malformations and metabolic disorders; another example was the identification of the NADPH oxidase activation with downstream events such as oxidative stress, cell cycle arrest, and apoptosis, which can result in adverse health effects, such as reduced sperm quality and quantity and disrupted folliculogenesis [14].

AOP concept can also be used for environmental risk assessment defining biomarkers that can be applied to estimate toxicity to aquatic organisms, as proposed Lee et al., (2015). The authors revised the relationship between environmental pollutants and toxic effects on fish exposed to benzo(a)pyrene, silver nanoparticules and selenium, and concluded that AOPs can be used for early environmental risk assessment. However, AOP approach should be carefully applied considering differences in the chemical susceptibility of distinct species [15].

Using the AOP framework, Khan et al. (2020) showed that early events on molecular levels can be connected to important effects on bivalves organisms, which can be used as relevant biomarkers for the assessment of environmental risk related to the presence of contaminants of emerging concern [16].

In this way, the AOP approach can be used as biomarkers of effects for different levels of organization, both for human health and environmental protection.

\section{In Silico Models: Balancing Mechanistic Interpretability, Predictivity, and Transparency}

In the context of in silico models, there is a recurrent discussion about the need for more transparency regarding the tools used and more efforts to solve the problem of "black boxes" models to facilitate its mechanistic interpretation $[17,18]$. To complicate this challenge, some transparent models can be less predictive than non-interpretable models.

There is a general assumption that category and alert-based models can have a lower accuracy and higher transparency and mechanistic interpretability than global statistical models $[17,18]$ because, depending on the method, it is possible to attribute activity for some fragments or generalizations for chemical categories (e.g., mutagenicity of aromatic amines). On the other hand, some statistical and global models can have generally a higher predictivity, considering the coverage of different structures 
and chemical categories present in datasets with a higher diversity of fragments and hypotheses. In this case, despite the loss of interpretability, the global model will learn more complex patterns generally not detected in more reduced chemical categories.

The choice of endpoints to predict by statistical and alert-based models using the AOP framework is an interesting way to balance mechanistic interpretability, predictivity, and transparency (Figure 3). By applying global statistical models using datasets of assays with a high mechanistic relevance (e.g., in vitro and/or in chemico) and strategies of mapping response-related fragments to different assays, it is possible to obtain predictions that are generally interpretable compared with other predictions along with the AOP framework.

in silico methods

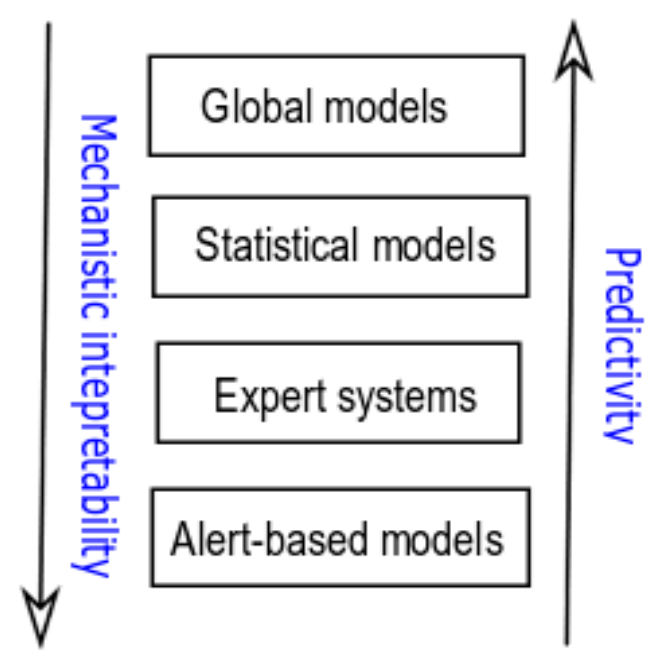

\section{Endpoint}

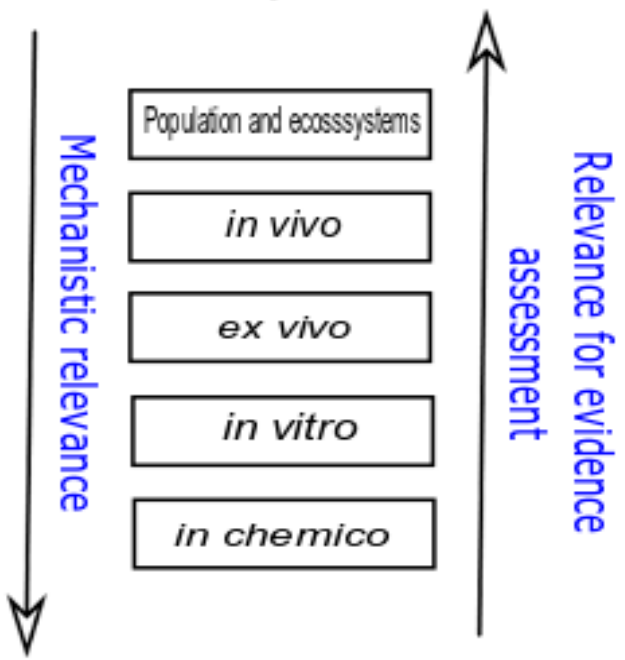

Figure 3. Mechanistic interpretability, predictivity, and transparency levels generally assumed for different in silico models and toxicological endpoints.

If measurable and plausible relationships between in chemico or in vitro endpoints with relevant in vivo or population outcomes are transparently predicted by the model, the result will add mechanistic interpretability and predictivity, facilitating scientific-based extrapolations and inferences that would add more evidence support and confidence to the regulatory decisions.

\section{Example of AOP-based In Silico Model}

During the Pan-American Conference for Alternative Methods in 2018, we proposed the concept for an "AOP-based in silico model", defined as "in silico framework used to identify chemicals that can activate the associated modular AOP components (MIE/KEs), and based in these individual multilevel predictions derived by in chemico, in vitro, ex vivo and in vivo data, balanced by adjustments, relationships, experimental data (if available) and weight of evidence assessment, to predict an adverse outcome".

Considering the opportunities of using this concept, recently we launched the In Silico Toxicology Platform (iS-Tox ${ }^{\circledR}$ ) (https://is-tox.com/our-models), with in silico tools free of charges for academic purposes and commercially available for private and regulatory purposes.

By integrating alerts (rule-based), statistical and machine learning-based models for metabolism, toxicity, and ecotoxicity endpoints, some tools use an adverse AOP framework [19], balancing transparency, mechanistic interpretability, and predictivity.

The tool AOP-Sens (Adverse Outcome Pathway-based model for Skin Sensitisation) is a computational tool that integrates in chemico, in vitro, and in vivo data in a targeted and predictive framework that simulates an Integrated Approach to Testing and Assessment (IATA) for skin sensitization $[20,21]$. The computational model is based on a combination of structural alerts and 
artificial intelligence (AI) models to predict responses for each key event: reactivity and Direct Peptide Reactivity Assay (DPRA) [22], KeratinoSens, human Cell Line Activation Test (h-CLAT), U-SENS, Local Lymph Node Assay (LLNA), and human sensitization [20,21]. As a final result, an AOP-based prediction is generated by the integration of individual models for the AOP components balanced by adjustments, Key Event Relationships (KERs), and Weight of Evidence (WoE) assessment (Figure 4). At the first level (superior side), we see the structure of the query chemical and some predicted physicochemical properties. At the second level, we see the prediction for the molecular initiating event (MIE); in this case, the model predicts protein reactivity by alerts (checking fragment known reactive to proteins mechanistically) and a statistical model derived from an experimental data DPRA assay. Metabolic activation is predicted by the metabolism model (prediction of metabolite structures, $\%$ of formation, and skin sensitization, not shown in this figure). The following levels are predictions for the KEs (cellular responses in the immunological and inflammatory responses to the progression of the defined biological perturbation) obtained by statistical models (with individual confidence scores and applicability domain-AD), leading to specific adverse outcomes. As a result, a positive AOP-based prediction was generated by the integration of individual models for the AOP components balanced by adjustments, Key Event Relationships (KERs), and Weight of Evidence (WoE) assessments. Interestingly, 3-Dimethylamino propylamine is known as a pro-hapten (not directly reactive before metabolic activation, but sensitizing after biotransformation), and the prediction of the AOP-based model supports this hypothesis. The complete report of this in silico assessment is provided as supporting material.

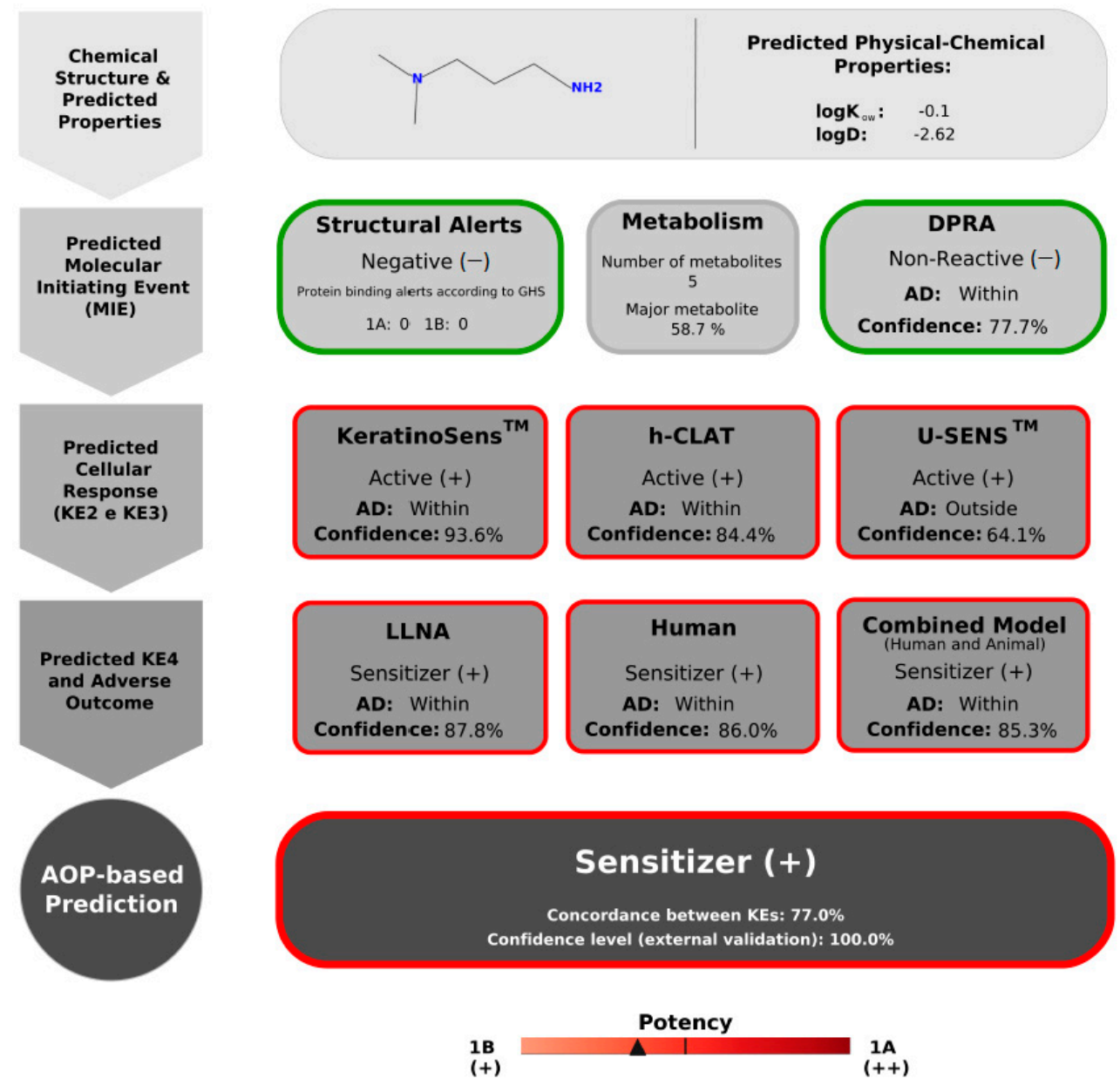

Figure 4. Example of an AOP-based in silico result generated by the AOP-Sens for the chemical 3-Dimethylamino propylamine (positive results are presented in red and negative results are presented in green). 
This is an example of an in silico model with a logical framework balancing transparency, mechanistic interpretability, and predictivity. The models are used to predict chemicals that can activate the AOP modular components (MIE/KEs), preserving mechanistic information, and a global model integrating all multilevel predictions balancing confidence scores, KERs and WoE adjustments, is used for predicting the adverse outcome.

As a perspective on using the $\mathrm{AOP}$ framework in quantitative approaches, in the last few years some authors have applied it for decision-making focusing on the transition from qualitative to quantitative AOP (qAOP) [19], and this transition is expected to be expanded to new toxicological endpoints.

\section{Setting and Connecting new biomarkers by in silico predictions and Adverse Outcomes Pathways (AOPs)}

There are many data published with biological responses in different levels of biological organization, and not appropriately reported by a hierarchical way or based on the AOP framework. Mechanistically relevant biomarkers of effect and/or exposure are probably not connected and are possibly hidden in literature and databases.

The major challenge and work for this topic is to translate this high volume of data into the AOP framework. After that, the second challenge is to extract information from this data generated from different protocols and contexts in order to establish networks and relevant biomarkers to different responses.

Finally, it is necessary to identify knowledge gaps concerning the mechanisms of action, key events, and causal associations with the outcomes observed in the target species (e.g., humans) that would be filled by in silico predictions and, if necessary, to establish criteria to apply this framework with regulatory purposes (practical applications).

Regarding these two last topics of the identification of gaps and connection between toxicological data or biomarkers for targeted outcomes and the establishment of criteria for regulatory applications, two selected works can be highlighted. Firstly, Baken et al. (2019) worked with an interesting strategy to validate a selection of human effect biomarkers using AOP, establishing connections between human observational studies and biological events involved in the toxicity pathways and mechanisms of action retrieved from the experimental studies and published AOPs [14]. Additionally, Patlewicz et al. (2019) proposed a scientific confidence framework (SCF) for evaluating and applying a given AOP for several different typical regulatory purposes, ranging from prioritizing chemicals to further risk assessments [23]. These works pointed out some examples of key events-biomarkers relationship and criteria for the assessment and application of structured AOPs. In the present work, some possible directions are proposed for integrating in silico models and Adverse Outcomes Pathways to fill gaps and setting and relating new biomarkers.

For the ecotoxicological context, some interesting cases of the application of the AOP framework to investigate potential changes on the population of aquatic species and to establish links between adverse response and biomarkers were reported by Fay et al. [24]. The authors assessed links between the known action of agonists or antagonists substances for different molecular receptors, such as ecdysone receptor (EcR), sodium-dependent serotonin transporter (SERT/5-HT), gamma-aminobutyric acid receptor (GABAr), Cyclooxygenase 1(COX1), and others, as well as outcomes on aquatic populations (reproductive impairment, population increase or decrease, mortality, etc.). Considering the high volume of chemicals that are known initiators of these specific pathways (mainly pharmaceuticals and biocides), the authors suggested the use of new biomarkers based on these receptors' activity, and additional investigations for the presence of these substances for the monitoring of potential environmental impact.

\section{Conclusions}

The AOP framework provides a structured and transparent means to organize the current data and all our knowledge about toxicity, generated by all types of assays (in chemico, in vitro, ex vivo, 
in vivo, and population studies). If the toxicological data is organized, new relationships between these data can be established and can generate new knowledge, improving our decisions. If we establish measurable and plausible relationships between some in silico, in chemico, or in vitro findings with relevant in vivo or population outcomes, a confidence level could be attributed for in silico, in chemico, and in vitro findings (including a set of selected biomarkers).

With this framework, many chemicals with any unknown in vivo or population outcomes could be efficiently and cost-effectively assessed by these data obtained by non-animal, low cost, and high-throughput methods. Additionally, the AOP framework would provide mechanistic reasoning for some chemicals with known in vivo or population outcomes but with no knowledge about the mechanisms, points of intervention, and any uncertainties. These scientific-based extrapolations and inferences would add more evidence support and confidence to the regulatory decisions.

Despite the challenges discussed in this work, by using the AOP framework to report ecotoxicological, toxicological, and structural data from in chemico, in vitro, ex vivo, in vivo, and population studies, it is expected that the resulting biological and chemical spaces will improve their rule as a knowledge platform to set and relate new biomarkers.

Depending on the endpoints being studied, as a well-stablished way or a perspective, some remarks and possibilities to explore the biological and chemical spaces could be highlighted:

- In exploring the biological space with the new AOPs generated, it will probably be possible to identify measurable key events without apparent significance individually, but with potential applicability when used as a set of biomarkers for the confirmation of active adverse pathways and/or exposures;

- In the reverse way, new hypotheses for molecular initiating events, key events, key event relationships, and/or adverse outcomes may be envisioned by known biomarkers or set of biomarkers;

- Exploring the chemical space, unknown chemicals can be assessed by in silico predictions or AOP-based in silico models based on known chemicals and structural similarity characteristics to predict metabolites, molecular initiating events, key events, key event relationships, and/or adverse outcomes, proposing potential pathways and biomarkers to be investigated;

- Exploring the chemical space, experimentally poorly assessed chemicals linked to adverse outcomes can be assessed by in silico predictions or AOP-based in silico models to predict molecular initiating events to fill gaps in toxicity pathways and to help in Weight of Evidence assessments with mechanistic inputs or predictive improvements by integration with biological data;

- Knowing success cases, it will be possible to establish criteria for setting new biomarkers and to apply these frameworks with regulatory purposes (practical applications).

Author Contributions: Conceptualization, C.E.M.d.S., D.P.d.O., and D.J.D.; Investigation, C.E.M.d.S., D.P.d.O., and D.J.D.; Resources, C.E.M.d.S., D.P.d.O., and D.J.D.; Writing—original draft preparation, C.E.M.d.S. and R.G.M.; Writing-review and editing, D.P.d.O. and D.J.D.; Supervision, D.P.d.O. and D.J.D.; Funding acquisition, C.E.M.d.S., D.P.d.O., and D.J.D. All authors have read and agreed to the published version of the manuscript.

Funding: This research was partially founded by São Paulo Research Foundation-FAPESP (grant number: 2016/08322-5). Additionally, this study was financed in part by the Coordenação de Aperfeiçoamento de Pessoal de Nivel Superior (CAPES)-Finance Code 001. We also thank to CAPES for the scholarship of RGM.

Acknowledgments: We thank São Paulo Research Foundation-FAPESP and Coordenação de Aperfeiçoamento de Pessoal de Nível Superior (CAPES) for all the assistance and funding. We also thank Altox for the software licenses, and all the associated partners, fellowships, and consultants that worked in the the iS-Tox Project: Rodolpho Braga; Iury Tércio Simões de Sousa; Luiz A. Mota Filho; Guilherme Yambanis Thomaz; Tiago Neiva Andrade; Carolina Horta Andrade (Universidade Federal de Goias / LabMol); Vinicius M. Alves (LabMol); Anderson Kamiya (Aka Technology).

Conflicts of Interest: The authors declare no conflict of interest. 


\section{References}

1. ICH M7. ICH M7-Assessment and Control of DNA Reactive (Mutagenic) Impurities in Pharmaceuticals to Limit Potential Carcinogenic Risk. Available online: https:/database.ich.org/sites/default/files/M7_R1_ Guideline.pdf (accessed on 5 October 2020).

2. USEPA-NAFTA TWG Quantitative Structure Activity Relationships [(Q)SAR] Guidance Document. 2012. Available online: https://archive.epa.gov/pesticides/news/web/pdf/qsar-guidance.pdf (accessed on 7 October 2020).

3. Rasinger, J.D.; Frenzel, F.; Braeuning, A.; Lampen, A. Identification and evaluation of potentially mutagenic and carcinogenic food contaminants. EFSA J. 2018, 16, e16085. [CrossRef] [PubMed]

4. Cotterill, J.V.; Chaudhry, M.Q.; Matthews, W.; Watkins, R.W. In silico assessment of toxicity of heat-generated food contaminants. Food Chem. Toxicol. 2008, 46, 1905-1918. [CrossRef] [PubMed]

5. EFSA PPR Panel (EFSA Panel on Plant Protection Products and their Residues). Guidance on the establishment of the residue definition for dietary risk assessment. EFSA J. 2016, 14, e04549. [CrossRef]

6. European Commission. Alternatives to Animal Testing and Safety Assessment of Chemicals. Available online: https://ec.europa.eu/jrc/en/research-topic/alternatives-animal-testing-and-safety-assessment-chemicals (accessed on 7 October 2020).

7. European Chemicals Agency. Practical Guide: How to Use and Report (Q)SARs. $2016.236 \mathrm{p}$. Available online: https://echa.europa.eu/documents/10162/13655/pg_report_qsars_en.pdf/407dff11-aa4a4eef-a1ce-9300f8460099 (accessed on 7 October 2020).

8. Hartung, T. Opinion versus evidence for the need to move away from animal testing. Altex 2017, 34, 193-200. [CrossRef] [PubMed]

9. OECD—Organisation for Economic Co-operation and Development. Revised Guidance Document on Developing and Assessing Adverse Outcome Pathways; Series on Testing \& Assessment, no. 184, Series on Adverse Outcome Pathways, No. 1; OECD: Paris, France, 2017.

10. OECD-Organisation for Economic Co-operation and Development. Users' Handbook Supplement to the Guidance Document for Developing and Assessing AOPs; Series on Testing \& Assessment, no. 233, Series on Adverse Outcome Pathways, No. 1; OECD: Paris, France, 2018; pp. 1-60.

11. Dan, V. Role of the Adverse Outcome Pathway Framework in the Validation of Predictive Biomarkers. Eurotox 2017. In Proceedings of the 53rd Congress of the European Societies of Toxicology, Bratislava, Slovakia, 10-13 September 2017; Available online: https://cfpub.epa.gov/si/si_public_record_report.cfm? Lab=NHEERL\&dirEntryId=340781 (accessed on 7 October 2020).

12. Sachana, M. Adverse Outcome Pathways and Their Role in Revealing Biomarkers; Elsevier: Amsterdam, The Netherlands, 2019; ISBN 9780128146552.

13. Spinu, N.; Bal-Price, A.; Cronin, M.T.D.; Enoch, S.J.; Madden, J.C.; Worth, A.P. Development and analysis of an adverse outcome pathway network for human neurotoxicity. Arch. Toxicol. 2019, 93, 2759-2772. [CrossRef] [PubMed]

14. Baken, K.A.; Lambrechts, N.; Remy, S.; Mustieles, V.; Rodríguez-Carrillo, A.; Neophytou, C.M.; Olea, N.; Schoeters, G. A strategy to validate a selection of human effect biomarkers using adverse outcome pathways: Proof of concept for phthalates and reproductive effects. Environ. Res. 2019, 175, 235-256. [CrossRef] [PubMed]

15. Lee, J.W.; Won, E.J.; Raisuddin, S.; Lee, J.S. Significance of adverse outcome pathways in biomarker-based environmental risk assessment in aquatic organisms. J. Environ. Sci. 2015, 35, 115-127. [CrossRef] [PubMed]

16. Khan, B.; Ho, K.T.; Burgess, R.M. Application of Biomarker Tools Using Bivalve Models Toward the Development of Adverse Outcome Pathways for Contaminants of Emerging Concern. Environ. Toxicol. Chem. 2020, 39, 1472-1484. [CrossRef] [PubMed]

17. Pavel, P. Interpretation of QSAR Models: Past, Present and Future. J. Chem. Inf. Model. 2017, 57. [CrossRef]

18. OECD-Organisation for Economic Co-operation and Development. Report of the Expert Consultation on Scientific and Regulatory Evaluation of Organic Chemistry Mechanism-Based Structural Alerts; Series on Testing and Assessment, No. 120 PART 1; OECD: Paris, France, 2010; pp. 1-85.

19. Spinu, N.; Cronin, M.T.D.; Enoch, S.J.; Madden, J.C.; Worth, A.P. Quantitative adverse outcome pathway (qAOP) models for toxicity prediction. Arch. Toxicol. 2020, 94, 1497-1510. [CrossRef] [PubMed] 
20. Patlewicz, G.; Simon, T.W.; Rowlands, J.C.; Budinsky, R.A.; Becker, R.A. Proposing a scientific confidence framework to help support the application of adverse outcome pathways for regulatory purposes. Regul. Toxicol. Pharmacol. 2015, 71, 463-477. [CrossRef]

21. OECD. Guidance Document on the Reporting of Defined Approaches and Individual Information Sources to Be Used within Integrated Approaches to Testing and Assessment (IATA) for Skin Sensitisation; OECD: Paris, France, 2016.

22. OECD. The Adverse Outcome Pathway for Skin Sensitisation Initiated by Covalent Binding to Proteins; OECD: Paris, France, 2014.

23. OECD. Draft Guideline for Defined Approaches for Skin Sensitisation. Available online: http://www.oecd. org/env/ehs/testing/GL\%20DASS_22Sep2019v2.pdf (accessed on 13 November 2020).

24. Fay, K.A.; Villeneuve, D.L.; LaLone, C.A.; Song, Y.; Tollefsen, K.E.; Ankley, G.T. Practical approaches to adverse outcome pathway development and weight-of-evidence evaluation as illustrated by ecotoxicological case studies. Environ. Toxicol. Chem. 2017, 36, 1429-1449. [CrossRef]

Publisher's Note: MDPI stays neutral with regard to jurisdictional claims in published maps and institutional affiliations.

(C) 2020 by the authors. Licensee MDPI, Basel, Switzerland. This article is an open access article distributed under the terms and conditions of the Creative Commons Attribution (CC BY) license (http://creativecommons.org/licenses/by/4.0/). 